\title{
A Novel Fabrication Process to Realise Piezoelectric Cantilever Structures for Smart Fabric Sensor Applications
}

\author{
Yang Wei, Russel Torah, Kai Yang, Steve Beeby and John Tudor \\ Electronics and Computer Science \\ University of Southampton \\ Southampton, U.K. SO17 1BJ \\ Email: yw6g08@ecs.soton.ac.uk
}

\begin{abstract}
This paper reports a novel process for realising freestanding piezoelectric cantilevers printed on fabric for potential use as a wearable sensor. Piezoelectric cantilever structures were fabricated by screen printing a piezoelectric stack on top of a structural layer that is supported by a sacrificial material during printing. The sacrificial layer was subsequently removed in an oven at $160{ }^{\circ} \mathrm{C}$ for 30 minutes which is sufficient to fully release the cantilever beam from the substrate. The samples were polarised by applying an electric field across the piezoelectric stack. Testing on an electromechanical shaker shows that the cantilevers have resonant frequencies at 390 and $260 \mathrm{~Hz}$ for beam lengths of 12 and $15 \mathrm{~mm}$, respectively. The beams produced maximum outputs of 38 and $27 \mathrm{mV}$ at an acceleration of $11.76 \mathrm{~m} / \mathrm{s}^{2}$, respectively.
\end{abstract}

\section{INTRODUCTION}

Smart fabrics aim to integrate electronic functions (e.g. sensors and actuators) into conventional fabrics offering new functionality. Many wearable electronics applications require smart sensors which are able to convert the physiological parameters or movement into electrical signals for data processing and evaluation. The piezoelectric effect is suitable to such applications because it is able to convert dynamic mechanical deformation into electric charge. Typically, piezoelectric strips/threads or films are woven into, or glued on top of, the fabric to form a sensor $[1,2]$. However, the woven approach restricts the sensor orientation and in both cases sensor performance is determined by the movement of the fabric itself.

Screen printing provides an alternative sensor fabrication technique. Screen printing uses a patterned screen to deposit functional materials onto fabrics, the placement and orientation of which is defined solely by the pattern. Therefore, the design has fewer design restrictions compared with other smart fabric fabrication techniques. In addition, screen printing is already an industry standard for creating coloured artwork on fabric and in printed electronics.

This paper demonstrates the feasibility of using screen printing to create piezoelectric free standing cantilever structures on fabric for smart sensor applications. Freestanding structures offer the potential for increased sensor performance since the piezoelectric material can be combined with a purpose designed mechanical structure located on the surface of the fabric.

\section{BACKGROUND}

Cantilever structures are used as the basis for a wide variety of MEMS sensors and actuators, such as accelerometers, resonators, and energy harvesters. These cantilever structures are normally fabricated using either bulk micromachining or surface micromachining. These fabrication processes typically require high temperatures, strong acids/alkalines and solvents.

Screen printing has also been used to fabricate free standing structures on top of a substrate using a printed sacrificial layer [3, 4]. S. Kok et.al. [5] demonstrated a PZT based freestanding cantilever structures on an alumina tile. The cantilever is made by screen printing a carbon based sacrificial layer followed by a combination of $\mathrm{Ag} / \mathrm{Pd}$ electrodes and PZT layers. The carbon sacrificial layer is removed during the firing process for the PZT layer $\left(850{ }^{\circ} \mathrm{C}\right.$ for 10 minutes). However, this fabrication process cannot be used for smart fabric applications because the high temperature will destroy the fabric. R. Lakhmi et al. [6] reported a force sensor created by screen printing. The sacrificial material is an epoxy loaded with strontium carbonate $\left(\mathrm{SrCO}_{3}\right)$; the structural material is a ceramic overglaze (ESL4702). The pastes require curing at $850{ }^{\circ} \mathrm{C}$ and the sacrificial material is removed by using phosphoric acid $\left(\mathrm{H}_{3} \mathrm{PO}_{4}\right)$ both of which are unsuitable for the majority of fabrics.

We previously reported a screen printed freestanding capacitive structure on fabric [3]. The piezoelectric sensors fabricated in this paper use this screen printable sacrificial material which can be dried at $80{ }^{\circ} \mathrm{C}$ to provide a solid platform for subsequent printed layers. This sacrificial material can then be removed in a box oven at $160{ }^{\circ} \mathrm{C}$ for 30 minutes. The process has been specifically designed to be compatible with most fabrics. The addition of a piezoelectric layer to the process will improve

This research is funded by EU project MICROFLEX(Grant No.: CP-IP 211335-2). 
sensitivity and enable simpler electronics than the previous capacitive approach.

There is a wide variety of alternative piezoelectric materials which could be used in this application. Piezoelectric materials exist in either crystal/polycrystalline forms (e.g. quartz and lead zirconate titanate (PZT)) or polymer form (e.g. PVDF) [7]. PZT is widely used for screen printing as it can be formulated into a ceramic paste whereas PVDF is typically spin coated onto a substrate. However, as PZT requires a high curing temperature $\left(>1400{ }^{\circ} \mathrm{C}\right)$ and produces rigid films, it cannot be used with fabrics directly. PVDF is difficult to screen print and is typically found in sheet form or in a liquid form with a too low a viscosity for screen printing. In addition, PVDF typically has a much lower piezoelectric response $(\sim 20 \mathrm{pC} / \mathrm{N})$ compared to PZT $(600 \mathrm{pC} / \mathrm{N})$.

A screen printable piezoelectric polymer was obtained from Meggitt [8]. This paste combines the advantages of ceramic PZT with the flexibility of a polymer. The curing process is $100{ }^{\circ} \mathrm{C}$ for 5 minutes which is compatible with fabrics. Using this material and the fabrication process described in [3], this work shows the fabrication of piezoelectric cantilever structures on fabrics.

\section{DEVICE DESIGN AND FABRICATION}

Fig 1 shows a drawing of the device. A screen printable sacrificial material based on the plastic crystalline Trimethylolethane (TME) was used for the sacrificial layer. This material starts to sublimate at a relatively low temperature $\left(83^{\circ} \mathrm{C}\right)$ with minimal mass loss. The sublimation rate increases exponentially as the temperature increases. This material is fully removed after 30 minutes at $160{ }^{\circ} \mathrm{C}$. A UV curable polymer (ElectraPolymers EFV4) and a heat curable silver paste (S-20) from Johnson Matthey were used as the structural and conductive materials respectively. The structural layer was cured for 30 seconds in a UV Light Technology cabinet and the silver was cured at $80{ }^{\circ} \mathrm{C}$ for 5 minutes to ensure minimal sublimation of the sacrificial layer. The Meggitt polymer PZT paste is printed on top of the structural material, followed by a top silver electrode) to convert the mechanical deformation to an electric signal. The dimensions of each sample are shown in Table 1. These dimensions were chosen to produce a mesoscale device to allow straightforward visual examination and handling of the device whilst evaluating the feasibility of the process.

Polyester/cotton $(65 \% / 35 \%)$ was selected as the fabric substrate because it is the most prevalent fabric for everyday clothing. Fabric presents a rough surface for printing which can reduce the print quality and performance of the subsequent printed pastes. Therefore, to reduce this surface roughness, an electrically insulating interface paste (Smart Fabric Inks Ltd Fabink-UV-IF1) was printed directly on the fabric to provide a smooth layer on top of the fabric in an area defined by the screen. Subsequently, the

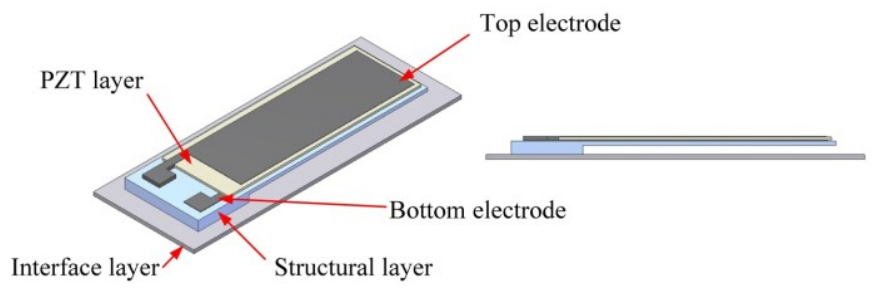

Figure 1. Schematic diagram of a piezoelectric cantilever (not to scale).

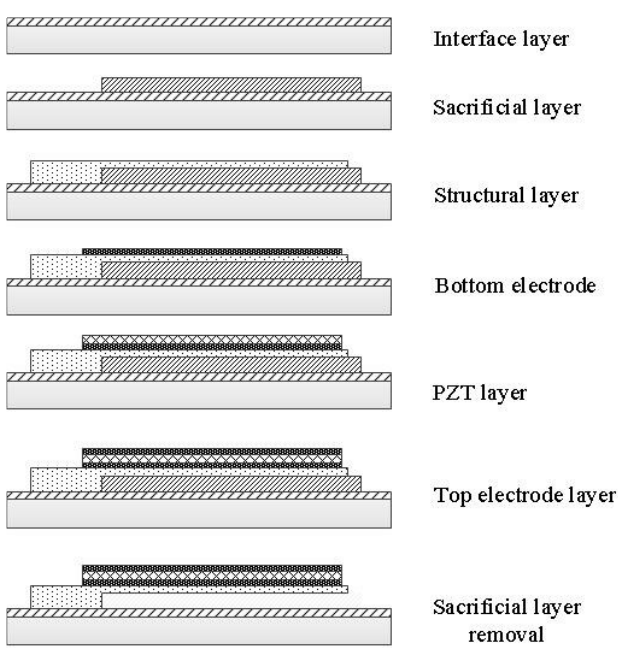

Figure 2. The fabrication sequence for a freestanding piezoelectric

\begin{tabular}{|c|c|c|}
\hline & Sample one & Sample two \\
\hline Electrode $(\mathrm{mm})$ & $11 \times 8$ & $14 \times 8$ \\
\hline $\operatorname{Beam}(\mathrm{mm})$ & $12 \times 10$ & $15 \times 10$ \\
\hline$P Z T(\mathrm{~mm})$ & $12 \times 10$ & $15 \times 10$ \\
\hline \multicolumn{3}{|c|}{ TABLE 2 Curing conditions of each layer. } \\
\hline Name & \multicolumn{2}{|c|}{ Curing conditions } \\
\hline Interface layer & \multicolumn{2}{|c|}{$U V$ radiation $1 \mathrm{~min}$} \\
\hline Sacrificial layer & \multicolumn{2}{|c|}{$80^{\circ} \mathrm{C} 5 \mathrm{~min}$} \\
\hline Structural layer & \multicolumn{2}{|c|}{$U V$ radiation $1 \mathrm{~min}$} \\
\hline Electrode layer & \multicolumn{2}{|c|}{$80^{\circ} \mathrm{C} 5 \mathrm{~min}$} \\
\hline PZT layer & \multicolumn{2}{|c|}{$100^{\circ} \mathrm{C} 5 \mathrm{~min}$} \\
\hline
\end{tabular}

sacrificial layer was printed and cured. The structural layer was then printed on the top followed by a Johnson Matthey S-20 polymer silver layer as the bottom electrode. The piezoelectric paste was printed on top of this electrode with a final top electrode printed to form a piezoelectric stack on top of the structural layer. The fabrication sequence and curing parameters of each layer are given in Fig 2 and Table 2. The curing requirements of these pastes are compatible with the sacrificial material and do not significantly reduce the mass loss of the sacrificial layer during the printing and curing of the subsequent layers.

Once all the devices were printed, the sacrificial layer was removed in a box oven at $160{ }^{\circ} \mathrm{C}$ for 30 minutes; example devices are shown in Fig 4. Visual examination confirmed that the sacrificial layer was completely removed. No sacrificial material residue remained and no significant sagging or bending of the structural layer was observed. Fig 3 shows an SEM cross sectional micrograph of a cantilever confirming that it has been fully released. The ragged edges observed on the structural layer in Fig 4 are due to some bleeding of the structural paste during printing. 


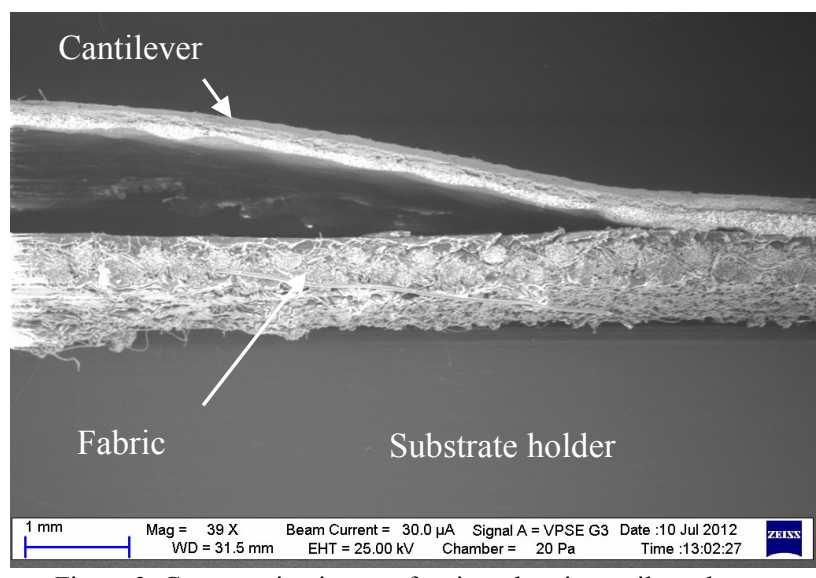

Figure 3. Cross section image of a piezoelectric cantilever beam.

\section{CANTILEVER TEST}

Wires were attached to each sample and the samples were polarised using a bespoke polarisation station. Once the samples were polarised, the piezoelectric coefficient $\left(\mathrm{d}_{33}\right)$ was measured using a PIEZOTEST PM300 piezometer; an average $d_{33}$ of 45 $\mathrm{pC} / \mathrm{N}$ was measured.

To evaluate the dynamic performance of the cantilever structure, a purpose built shaker rig was used to vibrate the sample whilst the output voltage from the PZT was measured. A charge amplifier is required to amplify the charge output from the PZT.

Samples were mounted onto the shaker plate and a frequency sweep was performed under a range of acceleration from 3.92 to $11.76 \mathrm{~m} / \mathrm{s}^{2}$. The results are shown in Fig 5 .

The mechanical resonant frequencies of two samples at an acceleration of $11.76 \mathrm{~m} / \mathrm{s}^{2}$ are $390 \mathrm{~Hz}$ and $260 \mathrm{~Hz}$ due to their different beam lengths $(12 \mathrm{~mm}$ and $15 \mathrm{~mm}$ ). Both samples show a

(a)

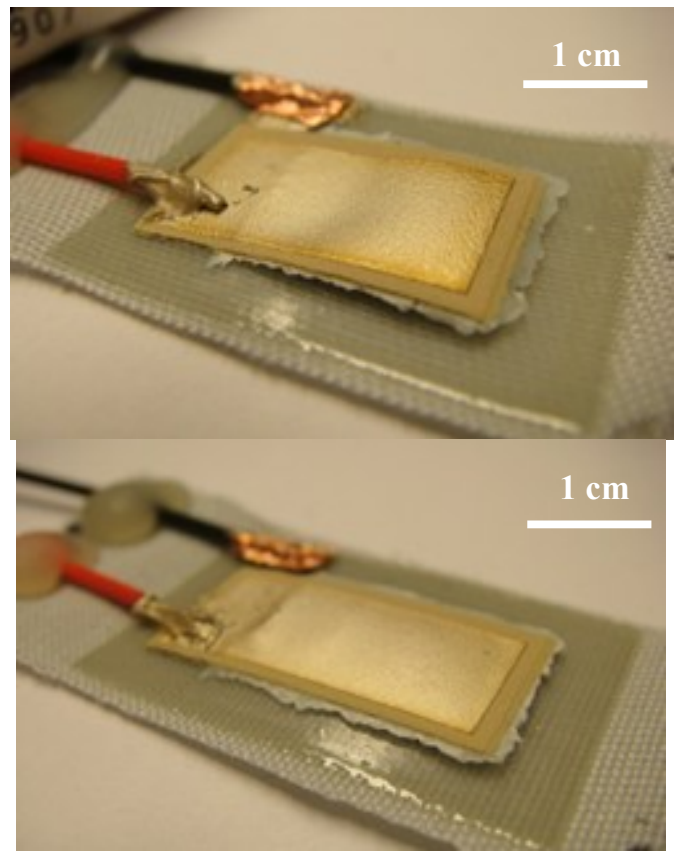

Figure 4. Piezoelectric cantilevers after the removal process: (a) sample one (L:12 mm); (b) sample two (L:15 mm).
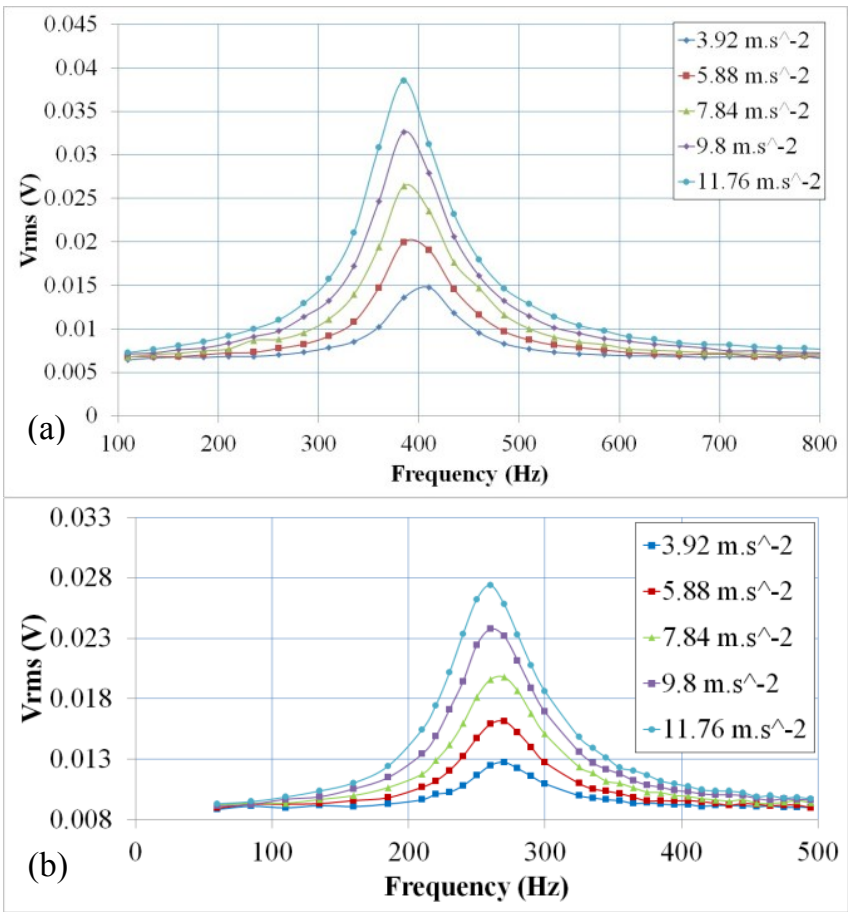

Figure 5. Output voltage versus frequency sweep: (a) sample one (L: 12 $\mathrm{mm}$ ); (b) sample two (L: $15 \mathrm{~mm}$ ).

soft non-linear response resulting in a slight decrease in resonant frequency with increasing amplitude of acceleration. This decrease is approximately $10 \mathrm{~Hz}$ on both samples. The maximum output voltages of two samples under an acceleration of 11.76 $\mathrm{m} / \mathrm{s}^{2}$ are $37 \mathrm{mV}$ and $27 \mathrm{mV}$, respectively.

The shaker was used in combination with a KEYENCE LC2450 laser displacement meter to measure the beam deflection over a range of frequencies. Displacements and voltage outputs for increasing and decreasing frequency sweeps were compared at an acceleration of $11.76 \mathrm{~m} / \mathrm{s}^{2}$. The results are shown in Fig 6 and, as expected, these show good agreement between voltage and displacement for both samples.

\section{CONCLUSIONS}

This paper has successfully demonstrated the feasibility of using a low temperature thermally removable sacrificial material TME and a piezoelectric polymer to fabricate piezoelectric cantilevers on a polyester/cotton $(65 \% / 35 \%)$ fabric. The sacrificial material can be screen printed and cured at $80{ }^{\circ} \mathrm{C}$ and can be completely removed in an oven at $160{ }^{\circ} \mathrm{C}$ for $30 \mathrm{~min}$. These temperatures do not produce any shrinkage or wrinkling of the fabric which confirms that this fabrication process is compatible with the fabric. The poling process for the piezoelectric polymer achieves an average $\mathrm{d}_{33}$ of $45 \mathrm{pC} / \mathrm{N}$ which compares very favourably with materials such as PVDF ( 20-30 $\mathrm{pC} / \mathrm{N}$ ) and remains compatible with the fabric. The harmonic test shows both samples have a soft non-linear response when the acceleration amplitude is increased resulting in a slight decrease in resonant frequency. The two samples with beam lengths of 12 $\mathrm{mm}$ and $15 \mathrm{~mm}$ have resonant frequencies of $390 \mathrm{~Hz}$ and $260 \mathrm{~Hz}$ respectively. 
(a)
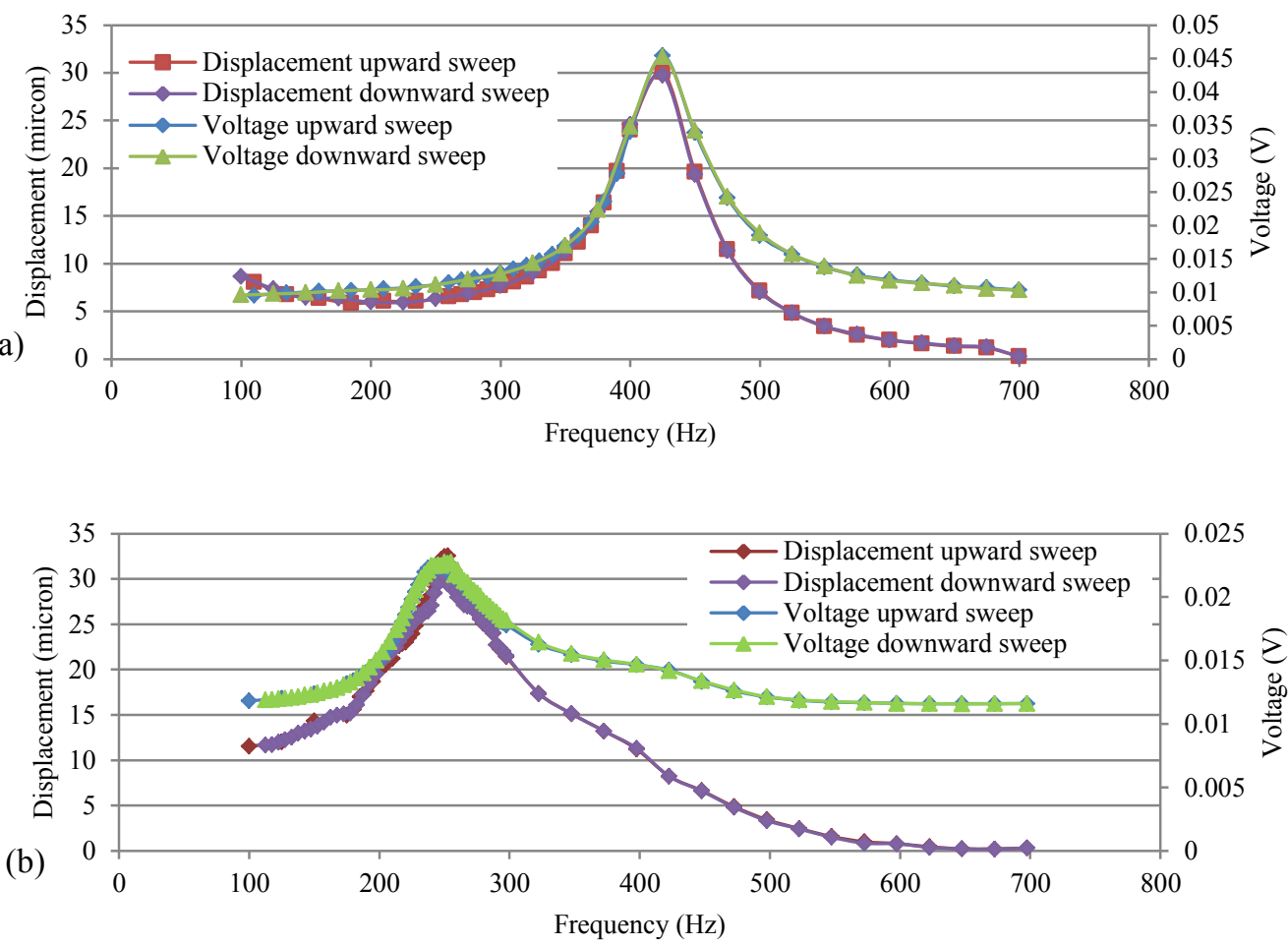

Figure 6. Displacement and voltage outputs from two samples: (a) sample one, L: $12 \mathrm{~mm}$; (b) sample two, L: $15 \mathrm{~mm}$.

The cantilever structure demonstrates the ability to fabricate a freestanding structure on fabric and therefore the technique could be used to make more complicated structures, such as accelerometers or pumps on fabric.

Future work will focus on reducing the size of the cantilever and increasing the print quality.

\section{ACKNOWLEDGEMENT}

The authors would like to acknowledge funding from the EU (Grant No.: CP-IP 211335-2) for the smart fabrics research project MICROFLEX (microflex.ecs.soton.ac.uk) under which this work was carried out. In addition, we would like to thank Klopman International for supplying the fabric, Smart Fabric Inks Ltd for supplying the screen printable interface paste, and MEGGITT A/S for supplying the screen printable piezoelectric polymer.

\section{REFERENCES}

[1] J. Edmison, M. Jones, Z. Nakad, and T. Martin, "Using piezoelectric materials for wearable electronic textiles," in 6th International Symposium on Wearable Computers, Washington D.C., USA, 2002.

[2] E. R. Post and M. Orth, "Smart fabric, or 'wearable clothing'," in 1st International Symposium on Wearable Computers, USA, 1997, pp. 167-168.

[3] Y. Wei, R. Torah, K. Yang, S. Beeby, and J. Tudor, "A novel fabrication process for capacitive cantilever structures for smart fabric applications," in Symposium on Design, Test, Integration \& Packaging of MEMS/MOEMS, Cannes, France, 2012.
[4] S. L. Kok, N. M. White, and N. R. Harris, "Free-standing thick-film piezoelectric device," Electronics Letters, vol. 44, pp. 280-282, 2008.

[5] S. L. Kok, "Design, fabrication and characterisation of free standing thickfilm piezoelectric cantilevers for energy harvesting," $\mathrm{PhD}$ thesis, School of Electronics and Computer Science, University of Southampton, 2010.

[6] R. Lakhmi, H. Debeda, I. Dufour, and C. Lucat, "Force sensors based on screen-printed cantilevers," IEEE Sensors Journal, vol. 10, pp. 1133-1137, 2010.

[7] T. Papakostas and N. White, "Screen printable polymer piezoelectrics," Sensor Review, vol. 20, pp. 135-138, 2000.

[8] Meggitt A/S. Available: http://www.meggitt.com/ 